\title{
Toxicological effects of graphene on mussel Mytilus galloprovincialis hemocytes after individual and combined exposure with triphenyl phosphate
}

\author{
Xiangjing Meng ${ }^{\mathrm{a}, \mathrm{c}}$, Fei Li ${ }^{\mathrm{a}, \mathrm{d}, *}$, Xiaoqing Wang ${ }^{\mathrm{a}, \mathrm{c}}$, Jialin $\mathrm{Liu}^{\mathrm{a}}$, Chenglong $\mathrm{Ji}^{\mathrm{a}, \mathrm{b}, \mathrm{d}}$, Huifeng $\mathrm{Wu}^{\mathrm{a}, \mathrm{b}, \mathrm{d}, * *}$ \\ ${ }^{a}$ CAS Key Laboratory of Coastal Environmental Processes and Ecological Remediation, Yantai Institute of Coastal Zone Research (YIC), Chinese Academy of Sciences \\ (CAS), Shandong Key Laboratory of Coastal Environmental Processes, YICCAS, Yantai 264003, PR China \\ ${ }^{\mathrm{b}}$ Laboratory for Marine Fisheries Science and Food Production Processes, Qingdao National Laboratory for Marine Science and Technology, Qingdao 266237, PR China \\ ${ }^{\mathrm{c}}$ University of Chinese Academy of Sciences, Beijing 100049, PR China \\ ${ }^{\mathrm{d}}$ Center for Ocean Mega-Science, Chinese Academy of Sciences, Qingdao 266071, PR China
}

\section{A R T I C L E I N F O}

\section{Keywords:}

Graphene

Triphenyl phosphate (TPP)

Joint effects

Immunity

Hemocytes

\begin{abstract}
A B S T R A C T
Graphene nanoparticles are increasingly released into the aquatic environment with the growth of production. However, there are rare investigations focusing on the interaction of nanoparticles with other contaminants. Triphenyl phosphate (TPP) is a frequently detected organophosphate flame retardant in the environment. This study aimed to assess the joint effects of graphene and TPP on Mytilus galloprovincialis hemocytes. Oxidative stress could be induced by graphene and TPP in mussel hemocytes, which could further cause apoptosis, DNA damage and decrease in the lysosomal membrane stability (LMS). Moreover, hemocytes could internalize graphene, thereby resulting in oxidative stress. The oxidative stress and DNA damage in hemocytes were increased in the graphene-exposed group, but significantly reduced after combined exposure of graphene and TPP. The upregulated genes, including $N F-\kappa B, B c l-2$ and Ras, were mainly associated with reduced apoptosis and DNA damage after co-exposure to graphene and TPP.
\end{abstract}

\section{Introduction}

At present, nanomaterials arouse global attention due to the potential inputs, risks of ecosystems and unpredictable damage to human health and environment (Gomes et al., 2013). Estuarine and coastal environments are considered as the ultimate sink areas of nanomaterials. The chemical behavior (aggregation/agglomeration) and consequent fate of nanomaterials may result in damage to marine ecosystems (Canesi et al., 2012). Graphene, a new material with extraordinary properties, attracts considerable concerns in various research communities and industries (Hu and Zhou, 2013; Iavicoli et al., 2017; Liu et al., 2016).

Due to the limitations of the detection method, there were few investigations on the environmental concentration of graphene. A previous study indicated that $>80 \%$ of graphene-family nanomaterials could be introduced into aquatic environments (Lanphere et al., 2014). Graphene-family nanomaterials might be transported by physical processes and deposited in the sediment (Chowdhury et al., 2013). Graphene could be transformed into graphene oxide (GO) in the natural environment under visible light irradiation (Hu et al., 2015). Hu et al. (2016) reported that the environmentally relevant concentration of GO was $1 \mathrm{mg} / \mathrm{L}$ in the aquatic environment.

With the development of ecological immunology, researchers attach more importance to the influence of environmental stressors on invertebrate immune defense mechanisms (Mezzelani et al., 2016; Politakis et al., 2018). When organisms are immunologically challenged, a series of immune responses may occur through various cell reactions, thereby resulting in phagocytic, cytotoxic or inflammatory responses (Ellis et al., 2011). Therefore, it is of great significance to explore how the invertebrate immune system responds to environmental changes (Mezzelani et al., 2016).

In recent years, the influence of graphene and its derivatives on marine invertebrate groups have aroused growing concerns (Canesi et al., 2015). For instance, immune and inflammatory responses were

\footnotetext{
* Correspondence to: F. Li, CAS Key Laboratory of Coastal Environmental Processes and Ecological Remediation, Yantai Institute of Coastal Zone Research (YIC), Chinese Academy of Sciences (CAS), Shandong Key Laboratory of Coastal Environmental Processes, YICCAS, Yantai 264003, PR China.

** Correspondence to: $\mathrm{H}$. Wu, Laboratory for Marine Fisheries Science and Food Production Processes, Qingdao National Laboratory for Marine Science and Technology, Qingdao 266237, PR China.

E-mail addresses: fli@yic.ac.cn (F. Li), hfwu@yic.ac.cn (H. Wu).
} 
shown in the hemocytes of mussels in the presence of commercial nanosized carbon black (NCB) (Canesi et al., 2008). The damage of immune system could be induced by nanoplastics accumulation in gills and hepatopancreas of Mytilus spp (Canesi et al., 2015). GO increased reactive oxygen species (ROS) production in $M$. galloprovincialis hemocytes at the highest sublethal concentration ( $25 \mathrm{mg} / \mathrm{L}$ ) tested (Katsumiti et al., 2017). It was also observed that mussels had highly developed nano- and micro-particle internalization mechanisms through endocytosis and phagocytosis, respectively (Moore, 2006).

As it was known, there could be multiple pollutants (such as heavy metals, organic pollutants, etc.) in natural environments, together with nanomaterials, such as graphene (Hu et al., 2014; Glomstad et al., 2016). It was reported that graphene could absorb organic contaminants through $\pi-\pi$ interactions (Wu et al., 2018; Sun et al., 2018). High levels of organic pollutants were increasingly released into the aquatic environment due to industrial activities and graphene could interact with these pollutants, which affected their joint toxicity to aquatic organisms (Al-Subiai et al., 2012; Meng et al., 2019).

As a potential substitute for brominated flame retardants (BFRs), triphenyl phosphate (TPP) has been widely used in many fields (Van der Veen and De Boer, 2012). TPP had multiplex toxicities, including neurotoxicity (Hong et al., 2018), developmental toxicity (Ding et al., 2017) and endocrine disrupting effects (Kim et al., 2015). The concentrations of TPP ranged from 7 to $209 \mathrm{pg} / \mathrm{g}$ dry weight (dw) in the surface sediments of the Bohai Sea and the Yellow Sea in China (Zhong et al., 2018). TPP has three benzene rings in its molecular structure, which makes TPP adsorb on graphene easily. Our previous work showed that combined graphene + TPP exposure could aggravate the damage of simulated phospholipid bilayer induced by single graphene or TPP exposure (F. Li et al., 2017; Y. Li et al., 2017). Based on these results, we speculated that combined exposure of graphene and TPP might induce differential toxic effects in marine organisms, compared with single exposure of graphene or TPP. Therefore, the joint toxic effects of graphene and TPP need to be further investigated.

Mussels Mytilus galloprovincialis, featured as wide distribution, filterfeeding activities, collection of particles from the seawater and phagocytic processes for cellular internalization of nanomaterials, are widely used for monitoring the marine environment (Balbi et al., 2017; Canesi et al., 2008; Canesi and Corsi, 2016; Pretti et al., 2014). Mussel has innate immune system, and its hemocytes are involved in phagocytosis or encapsulation (Dallas and Jha, 2015; Di et al., 2011). However, there were rare investigations on the potential toxicity of graphene-family nanomaterials combined with organophosphate flame retardant in mussels either in vivo or in vitro. This work aimed to explore the cellular toxicity of graphene and TPP in marine M. galloprovincialis.

In this study, the activities of catalase (CAT), superoxide dismutase (SOD) and glutathione-s-transferase (GST) and the content of GSH, were examined to elucidate the oxidative stress of graphene and TPP in M. galloprovincialis hemocytes. Then, the apoptosis rates of hemocytes were counted and the immune functions of hemocytes were estimated based on changes in the lysosomal membrane stability (LMS) and phagocytic activity. In order to measure in vitro genotoxicity, micronucleus (MN) assay (a biomarker of effect) was adopted in this study. Furthermore, to explore the hemocyte damage and the uptake of graphene, the ultrastructure of the hemocytes was measured. Levels of mRNA expression involved in apoptosis and immunity were also quantified to evaluate the effects of graphene and TPP at molecular level. In the end, integrated biological response (IBR) index was calculated to measure risks to the environment, and the toxic effects of graphene, TPP and graphene + TPP on mussel hemocytes were compared.

\section{Materials and methods}

\subsection{Chemicals and characteristics of graphene}

TPP (CAS No. 115-86-6) was purchased from J\&K Chemical Co., Ltd. Graphene nanomaterial was obtained from Sigma-Aldrich (Shanghai, China). The size and shape of graphene were characterized using atomic force microscopy (AFM, Veeco Multimode $8+$ bioscope catalyst, USA). The dynamic light scattering (DLS) with a ZetaPALS instrument (Malvern, Zeta sizer Nano Sizer, Nano-2S90, U.K.) was used to detect the size and charge distribution of graphene. Graphene stock dispersion $(200 \mu \mathrm{L})$ was initially freeze-dried into graphene powders, which were then evaluated using a DXR Raman spectrophotometer (Thermal Fisher, USA) with a $633 \mathrm{~nm}$ laser source.

\subsection{Experimental animals and conditions}

Adult M. galloprovincialis (4-5 $\mathrm{cm}$ in size) were purchased from a local culturing farm (Yantai, China). These animals were transported to the laboratory and acclimatized in aerated natural seawater (30 psu, $18 \pm 1{ }^{\circ} \mathrm{C}$ ) for $7 \mathrm{~d}$. Our previous work showed that graphene and TPP at $0.5 \mathrm{mg} / \mathrm{L}$ could influence the permeability of cell membrane and damage the integrity of the simulated phospholipid bilayer (F. Li et al., 2017; Y. Li et al., 2017). The concentration of graphene in this work was selected depending on the environmental concentration predicted at levels of 0.001-1 mg/L (Y. Zhang et al., 2017; X. Zhang et al., 2017; Hu et al., 2016). For TPP, the $E C_{50}$ values of the marine invertebrate were $0.26-2.0 \mathrm{mg} / \mathrm{L}$ (Liu et al., 2012). Therefore, the concentration of TPP in this work was designed at $0.5 \mathrm{mg} / \mathrm{L}$. For the experiments, the acclimated mussels were randomly divided into four groups: (i) seawater (control); (ii) $0.5 \mathrm{mg} / \mathrm{L}$ graphene; (iii) $0.5 \mathrm{mg} / \mathrm{L} \mathrm{TPP}$; (iv) $0.5 \mathrm{mg}$ / L TPP and $0.5 \mathrm{mg} / \mathrm{L}$ graphene. The seawater was changed daily and constantly aerated to ensure animal oxygen supply during acclimation and experimental periods. Each mussel was fed daily with approximately $30 \mathrm{mg}$ of dry-Chlorella vulgaris $(30 \mathrm{mg} / \mathrm{mussel})$. No mortality was observed during the experiment conducted in the laboratory.

\subsection{Hemolymph collection and handling}

After exposure for $7 \mathrm{~d}$, a sterile syringe $(1 \mathrm{~mL})$ without needle, containing $0.5 \mathrm{~mL}$ of anti-aggregation solution $(20.8 \mathrm{~g} / \mathrm{L}$ glucose, $8 \mathrm{~g} / \mathrm{L}$ sodium citrate, $3.36 \mathrm{~g} / \mathrm{L}$ EDTA, $22.5 \mathrm{~g} / \mathrm{L} \mathrm{NaCl}, \mathrm{pH}$ 7.0), was used to extract an amount of $500 \mu \mathrm{L}$ of hemolymph from the posterior adductor of each mussel. Then, the hemolymph was immediately centrifuged at $1500 \mathrm{rpm}$, for $5 \mathrm{~min}$ at $4{ }^{\circ} \mathrm{C}$, followed by the collection of supernatant (serum) and cell pellet (isolated hemocytes). The supernatant was used for examining antioxidant status (GST, SOD, CAT and GSH), while the hemocytes were used for cytotoxicity analysis (DNA damage, LMS and phagocytic activity, etc.).

\subsection{Evaluation of lysosomal membrane stability}

Following the previous study, a specific method was used to detect the LMS (Pipe et al., 1999). A total of $500 \mu \mathrm{L}$ of suspensions, containing $50 \mu \mathrm{L}$ of $0.33 \%$ neutral red solution in PBS ( $2 \% \mathrm{NaCl}, \mathrm{pH} 7.4)$, was incubated at $10{ }^{\circ} \mathrm{C}$ for $1 \mathrm{~h}$. The cells were then centrifuged at $300 \mathrm{~g}$ for $10 \mathrm{~min}$ and washed twice using filtered seawater (FSW). The $500 \mu \mathrm{L}$ of aliquots ( $1 \%$ acetic acid: $50 \%$ ethanol) was added in the suspensions, which was then incubated in darkness at $20{ }^{\circ} \mathrm{C}$ for $15 \mathrm{~min}$. The absorbance of the extraction solution was detected at $550 \mathrm{~nm}$ on a spectral scanning multimode reader (Tecan Austria GmbH 5082 Grodig, Austria). The data were expressed as $\mathrm{OD}_{550}$ (optical density) per $\mathrm{mg}$ hemocyte protein. Determination of protein concentration was performed using the BCA method (two cinchonine determination) (Della Torre et al., 2015). 


\subsection{Measurement of apoptosis}

An annexin V-fluorescein isothio-cyanate (FITC)/propidium iodide (PI) apoptosis detection kit (BD Biosciences, USA) was used to detect cell apoptosis. The cells were repeatedly washed for three times using ice-cold PBS. Following the instruction of the manufacturer, $10 \mu \mathrm{L}$ of annexin-V FITC and $5 \mu \mathrm{L}$ of PI stock solution were used for cell staining for $15 \mathrm{~min}$. After incubation at $4{ }^{\circ} \mathrm{C}$ for $15 \mathrm{~min}$ without light, cells were analyzed using a flow cytometer (FCM) (NovoCyte 1040, ACEA Biosciences, USA) following the standard instructions.

\subsection{Phagocytic activity}

The percentage of phagocytic cells refers to the percentage of cells internalized as one or more fluorescent beads in cells (Jiang et al., 2017). FCM was used for analyzing the ingestion of fluorescent beads. A volume of $30 \mu \mathrm{L}$ of fluorescent beads was dissolved in $1.5 \mathrm{~mL}$ of FSW for preparing the dilution of fluorospheres, followed by the incubation of $200 \mu \mathrm{L}$ of hemocyte suspension and $50 \mu \mathrm{L}$ of bead dilution at room temperature without light for $1 \mathrm{~h}$. An equal volume of Baker's Formol Calcium was used to stop the reaction.

\subsection{Antioxidant status}

The cell supernatant (serum) was collected after exposure for $7 \mathrm{~d}$. The commercial kits were used to examine the activities of GST, SOD, CAT and the content of GSH from serum, following the instructions of the manufacturer (Nanjing Jiancheng Bioengineering Institute, China). A spectral scanning multimode reader was used to record the absorbance at $490 \mathrm{~nm}$ (Tecan Austria GmbH 5082 Grodig, Austria).

\subsection{Quantitative real-time polymerase chain reaction (PCR)}

The TRIzol reagent was used to extract the total RNA from cells (Invitrogen, USA). The first-strand cDNA was synthesized following the usage information (Promega, USA). The expression levels of apoptosis and immune-related genes were presented in an Applied Biosystem 7500 Real-time PCR System, with gene primers listed in Table 1. $\beta$-actin was used as an internal standard, and the comparative $\mathrm{Ct}$ method with the formula $2^{-\triangle \triangle \mathrm{CT}}$ was chosen for calculating the relative gene expression levels (Livak and Schmittgen, 2001). SYBR ${ }^{\circledR}$ Select Master Mix kit was used for the standard qRT-PCR (Life Technologies, USA) on an ABI 7500 SDS software (Applied Biosystems, USA).

\subsection{Determination of micronucleus frequency}

The determination method of $\mathrm{MN}$ was based on the procedures described by Bolognesi and Fenech (Bolognesi and Fenech, 2012). For cell adhesion, the slides with suspensions were incubated for $15 \mathrm{~min}$ in the humid environment, and immersed in anhydrous methanol for $10 \mathrm{~min}$. Then these slides were dried and fixed in fume hood at room temperature. Giemsa solution $(3 \%$, v/v) was used to stain slide for $5 \mathrm{~min}$, followed by twice rinsing in washing solution $(2 \%$ sorensen buffer, made up by $53.4 \%$ solution $\mathrm{A}\left(9.073 \mathrm{~g} / \mathrm{L} \mathrm{KH}_{2} \mathrm{PO}_{4}\right)$ and $46.6 \%$ solution B (11.87 g/L Na $\left.\mathrm{HPO}_{4} \cdot 2 \mathrm{H}_{2} \mathrm{O}\right)$ ) for 5 min. Eukitt (FLUKA, Sigma-Aldrich, USA) was used for sealing and drying slides at room temperature prior to the observation under the $400 \times$ magnification of light microscope (Eclipse Ni-U, Nikon, Japan). To measure the frequency of micronuclei (MNi), a minimum of 1000 cells were randomly collected from each slide. Only cells with intact cellular and nuclear membrane were scored. MNi were round or oval in shape and characterized with the presence of a main nucleus and one or more smaller nuclei (Y. Zhang et al., 2017; X. Zhang et al., 2017).

\subsection{TEM analysis}

For transmission electron microscope (TEM) analysis, a sterile syringe ( $1 \mathrm{~mL}$ ) without needle containing $0.5 \mathrm{~mL}$ of $2.5 \%$ glutaraldehyde solution, was used to extract an amount of $500 \mu \mathrm{L}$ of hemolymph from the posterior adductor of each mussel. Then, the hemolymph was immediately centrifuged at $1500 \mathrm{rpm}$, for $5 \mathrm{~min}$ at $4{ }^{\circ} \mathrm{C}$, followed by removing the supernatants. The harvested cells were fixed with $2.5 \%$ glutaraldehyde solution and embedded in epoxy resin. The ultrathin cell specimens $(70 \mathrm{~nm})$ were placed on the grids and stained using $1 \%$ lead citrate and $0.5 \%$ uranyl acetate. A high-resolution TEM was used for the analysis on cells (JEOL JEM 2010F, Hitachi Scientific Instruments, Japan).

\subsection{Integrative biomarker indices}

Six biomarkers, including MN, LMS, CAT, SOD, GSH and GST were used for IBR calculation. The IBR value was based on the calculation of triangular star plot areas for each two neighboring biomarkers in a given data set (Xie et al., 2016). The procedures were described below (Xie et al., 2016):

(1) the mean and standard deviation (S.D.) was calculated for each biomarker of sampling treatments;

(2) the data were standardized for each treatment: $A=(B-a) / b$,

where A means the standardized value of biomarker, B denotes the value of biomarker responses, $a$ was the mean value of biomarker, and $b$ refers to the standard deviation of biomarker.

The IBR values were calculated using Beliaeff and Burgeot's method, while the scores of biomarker were visualized using a star plot where the radial coordinate was in accordance with the score (Beliaeff and Burgeot, 2002).

\subsection{Statistical analysis}

The enzyme activities and gene expressions in the samples of hemocytes, were expressed as means $\pm S$.D. The normality (KolmogorovSmirnov test, $P<0.05)$ and homogeneity of variances were tested using all data available (Levene's test, $P<0.05$ ). Based on the one-way analysis of variance (ANOVA) using SPSS 22.0 software, and Tukey's test, the differences between control and treated cells were investigated. The $P$ value $<0.05$ was considered statistically significant.

Table 1

The list of primers used for the determination of gene expressions by qRT-PCR in this study.

\begin{tabular}{|c|c|c|c|}
\hline Gene name & Accession No. & Forward primer $\left(5^{\prime}-3^{\prime}\right)$ & Reverse primer $\left(5^{\prime}-3^{\prime}\right)$ \\
\hline Bcl-2 & КС545829.1 & GTTAGACTTGCCACCATCA & CAGTAGCCTCAGTTATGTGTA \\
\hline Ras & DQ305041.1 & GCAGGACAGGAGGATTATGC & CCCAACCAACAGAAAAGGAA \\
\hline$N F-k B$ & KF051275.1 & ATACCTCTTCCGTCCGCACTT & AAGAAGCTGCCGCTAAATCGA \\
\hline Caspase 2 & HQ424449.1 & GATATATGACAAGGGTGGCAATG & GACTTTACAGCATCCAGGACATC \\
\hline Caspase 8 & HQ424450.1 & CCCAACCAGTAGTAACACCA GAC & GTATGAACCATGCCCCTATA TCA \\
\hline Caspase 3 & EF080944.1 & GATCTTGGAAGTGGTGTAGA ACG & CACTGCTAGGAAATCTGCTT CAT \\
\hline
\end{tabular}

Abbreviations: $B c l-2$, B-cell lymphoma-2; $N F-k B$, nuclear factor kappa-B. 
A

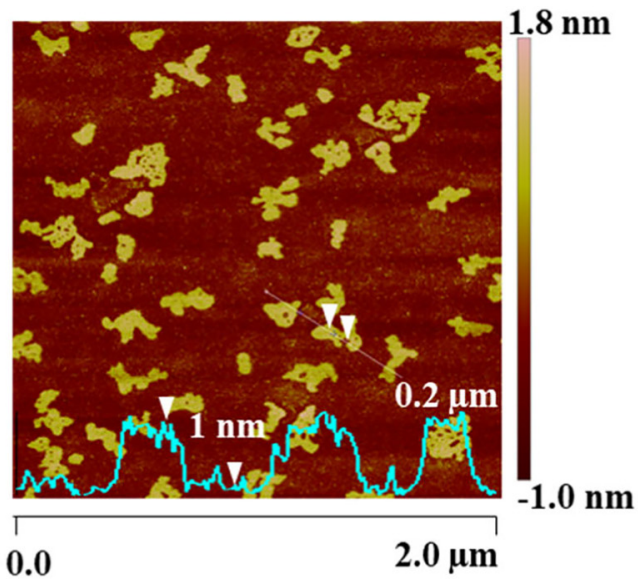

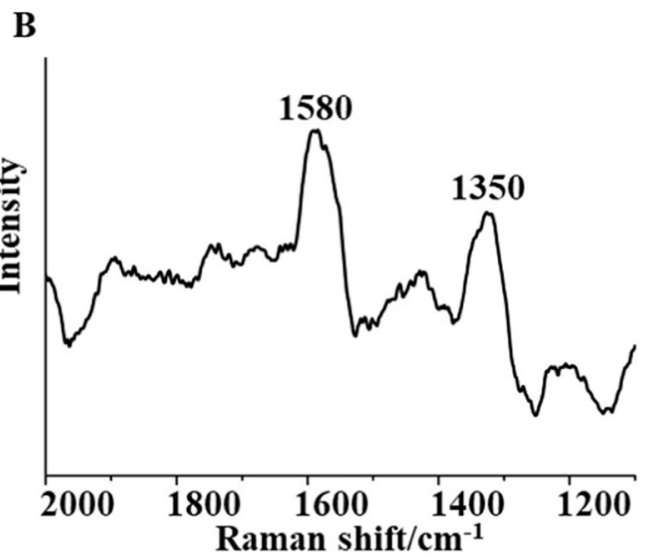

D

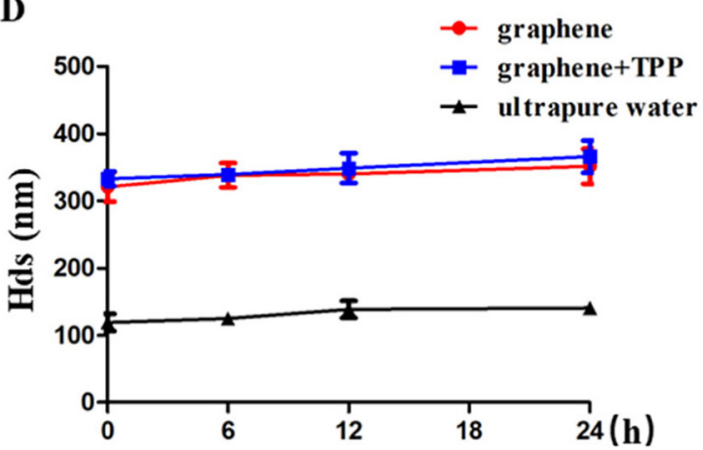

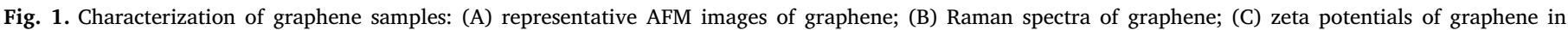

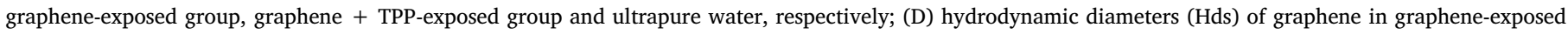
group, graphene + TPP-exposed group and ultrapure water, respectively.

\section{Results and discussion}

\subsection{Characterization of graphene}

The nanomaterial morphology of graphene was analyzed using AFM. Typical nanosheet morphology was observed in the employed graphene. As shown in Fig. 1A, the graphene sheet had a thickness of $1.0 \mathrm{~nm}$, which was consistent with the thickness $(\sim 1.0 \mathrm{~nm})$ of a single layer of graphene (Qiu et al., 2014), and the lateral lengths ranged from about 0.1 to $0.3 \mu \mathrm{m}$. The Raman spectra of graphene revealed the presence of typical D and G peaks at 1350 and $1580 \mathrm{~cm}^{-1}$, respectively (Fig. 1B) (Y. Zhang et al., 2017; X. Zhang et al., 2017).

As shown in Fig. 1C and D, the zeta potentials and Hds of graphene in the ultrapure water from 0 to $24 \mathrm{~h}$ were around $-30 \mathrm{mV}$ and $120 \mathrm{~nm}$, respectively. The zeta potentials and Hds values of graphene were around $-10 \mathrm{mV}$ to $-11 \mathrm{mV}$ and $320-350 \mathrm{~nm}$ in the graphene and graphene + TPP groups ( 0 h, 6 h, 12 h and 24 h), respectively. These results suggested that the graphene had agglomeration behavior in both graphene- and graphene + TPP-treated groups due to the high salinity of seawater. However, the zeta potentials and Hds values of graphene did not change significantly during the $0-24 \mathrm{~h}$ exposure. Therefore, the comparative toxicity on $M$. galloprovincialis hemocytes for the graphene and graphene + TPP treatments should be reasonable based on the characterization results of graphene.

\subsection{Antioxidant response to graphene, TPP and graphene + TPP exposures}

The role of contaminants in disrupting the balance between the ROS production and the antioxidant defense can be measured using biomarkers associated with oxidative status (Brandts et al., 2018). As shown in Table 2, there were significant increases in the activities of GST and CAT and the content of GSH $(P<0.05)$ in mussel hemocytes
Table 2

Antioxidant system response to graphene and TPP exposures in the present study*.

\begin{tabular}{lllll}
\hline Groups & Control & Graphene & TPP & Graphene + TPP \\
\hline SOD & $7.49 \pm 0.79$ & $6.93 \pm 0.93$ & $7.76 \pm 0.53$ & $7.15 \pm 1.32$ \\
GST & $8.87 \pm 1.61$ & $14.27 \pm 0.72^{\mathrm{a}}$ & $9.84 \pm 1.35$ & $9.72 \pm 3.07^{\mathrm{b}}$ \\
CAT & $1.81 \pm 1.46$ & $3.12 \pm 0.54^{\mathrm{a}}$ & $3.01 \pm 0.57^{\mathrm{a}}$ & $1.77 \pm 0.12^{\mathrm{b}, \mathrm{c}}$ \\
GSH & $4.41 \pm 0.89$ & $6.05 \pm 1.16^{\mathrm{a}}$ & $4.48 \pm 1.80$ & $6.30 \pm 2.08^{\mathrm{a}}$ \\
\hline
\end{tabular}

* The data are presented as the mean \pm S.D.

a $P<0.05$, compared with the control group.

b $P<0.05$, compared with the graphene-exposed group.

c $P<0.05$, compared with the TPP-exposed group.

after graphene exposure. In the TPP-exposed group, the CAT activity increased significantly $(P<0.05)$. The antioxidants play an important role in protecting bivalves against the negative effects of oxidative stress (Matozzo et al., 2016). The oxidative stress in vitro and in vivo could be enhanced by graphene-based nanomaterials, which might further cause cells apoptosis (Mittal et al., 2016; Zhu et al., 2017). As reported by Chen et al. (2015), the treatment with TPP could enhance the activities of GPX, SOD and CAT, and the transcriptional levels of their respective genes. Our findings were consistent with previous studies, indicating that both TPP and graphene could induce oxidative stress in M. galloprovincialis hemocytes.

Compared with graphene-exposed group, however, the activities of GST and CAT decreased significantly in the graphene + TPP-exposed group $(P<0.05)$, indicating that the oxidative stress was reduced after graphene + TPP treatment. Graphene could absorb organic contaminants such as polycyclic aromatic hydrocarbons (PAHs), organochlorine pesticides (OCPs) and polybrominated diphenyl ethers (PBDEs) (Wu et al., 2018). There were strong $\pi-\pi$ interactions between 
A

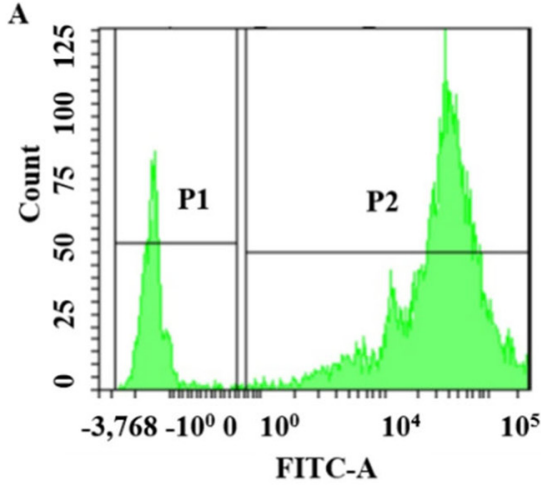

C

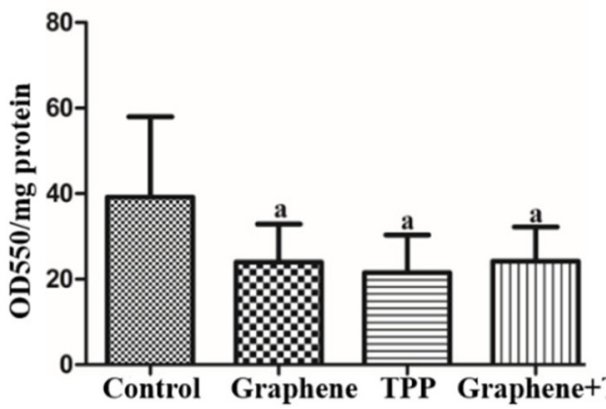

B

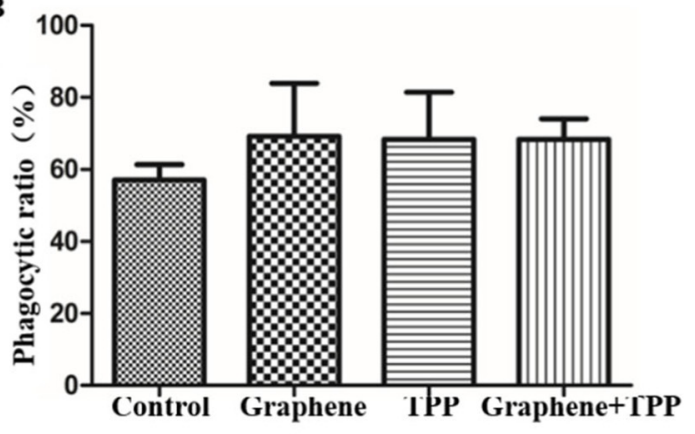

D

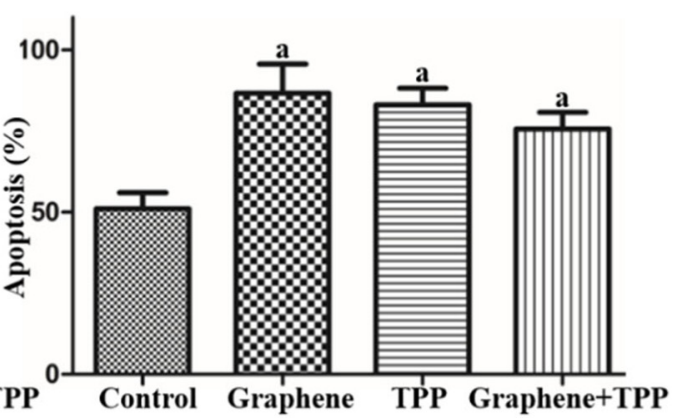

Fig. 2. The cytotoxicity of graphene and TPP. (A and B) Phagocytic of hemocytes; (C) lysosomal membrane stability; (D) hemocytes apoptosis. Note: (A) P1 was the hemocytes phagocytizing no beads, P2 was the hemocytes phagocytizing one or more beads.

graphene and PAHs molecules on the surface of spinach leaves (Sun et al., 2018). TPP had three benzene rings in its molecular structure, which made TPP adsorb on graphene easily. In our previous study, the average TPP concentration in graphene + TPP-exposed group was higher than that in the TPP-exposed group (about 1.9 times) (Meng et al., 2019). Therefore, the surface activity of graphene was inhibited with the adsorption of TPP on graphene, which therefore reduced graphene-induced oxidative stress in mussel hemocytes.

\subsection{Assessment of cytotoxicity induced by graphene, TPP and graphene +} TPP

Oxidative stress might cause apoptosis, cell membrane damage and other toxic effects (Canty et al., 2009; Ou et al., 2016). Compared with the control group, the phagocytic abilities slightly increased in all treatments without statistical significance (Fig. 2B). However, LMS and apoptosis rates of mussel hemocytes decreased and increased significantly $(P<0.05)$ in all treatments, respectively (Fig. 2C and D). Two main types of nuclear abnormalities ( $\mathrm{MN}$ and binucleated cells) were observed in mussel hemocytes (Fig. 3A). MNi frequencies increased in hemocytes in all treatments (Fig. 3B). Compared with the graphene-treated mussels, the MNi frequencies decreased in graphene + TPP-exposed mussels $(P<0.05)$.

Biomarkers are useful for monitoring pollution, and LMS is considered as a reliable indicator of general stress in marine organisms (Coteur et al., 2005; Moore, 2008). In this work, both graphene and TPP caused significant reductions of LMS (Fig. 2C), reflecting the harmful effect of graphene and TPP in mussels' immune system. A previous study found that nano- $\mathrm{TiO}_{2}$ could also induce a significant decrease in LMS (55\%) in mussel M. galloprovincialis (Balbi et al., 2014), which were consistent with our findings.

Apoptosis is closely correlated with the oxidative stress induced by environmental pollutants (Koutsogiannaki et al., 2015). In mollusk hemocytes, apoptosis was reported as a general cellular immune response (Cao et al., 2018; Romero et al., 2015). In this work, the increase in $(P<0.05)$ apoptosis was observed, due to graphene and TPP exposure. Similarly, GO or reduced graphene oxide (rGO) decreased cell adhesion and induce cell apoptosis in normal human lung cells (Vallabani et al., 2011). However, there was no significant difference between graphene and graphene + TPP treatments.

There was a negative correlation between LMS and efficiency of DNA damage in Mytilus cells (Balbi et al., 2018). MN assay for MNi frequency detection was the most popular and promising technique for examining genotoxic effects of single cells (Kolarevic et al., 2016). MN assay showed that graphene and TPP could induce DNA damage in mussel hemocytes. Interestingly, nano- $\mathrm{TiO}_{2}$ could increase the frequency of MNi in mussel cells as well (Della Torre et al., 2015). Compared with graphene, graphene + TPP could reduce the MNi frequency, suggesting an antagonistic effect in DNA damage between graphene and TPP in mussel hemocytes.

\subsection{Effects of graphene and TPP exposures on gene expression}

To gain insights into the mechanisms of the combined effects of graphene + TPP on M. galloprovincialis hemocytes, the expressions of apoptosis and immune-related genes were quantified. The relative expression of $N F-\kappa B$ gene in TPP-exposed group was evidently downregulated in comparison with the control group. For graphene-exposed group, the relative expressions of caspase2, caspase 3 and caspase 8 proapoptotic genes were significantly up-regulated $(P<0.05)$ (Fig. 4). Compared with graphene-exposed group, these genes were significantly down-regulated after the graphene + TPP exposure. The immune-related and blocking apoptosis genes, $N F-\kappa B, B c l-2$ and Ras, presented significantly higher expression levels in mussel hemocytes after graphene + TPP exposure, compared with those in both control and graphene treatment (Fig. 4).

Caspases are key molecules involved in death signaling and responsible for many apoptosis-related biochemical and morphological changes (F. Li et al., 2017; Y. Li et al., 2017). The caspase signaling pathway was triggered by oxidative stress in graphene and TPP-treated mussels, accompanied by the activation of caspase 2 and caspase8, two proapoptotic members of the caspase protein family (Cao et al., 2018). 
A

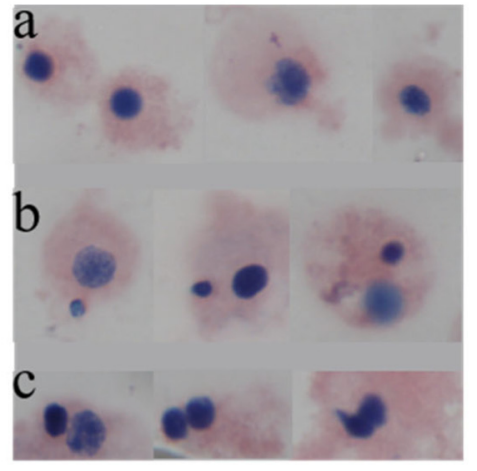

B

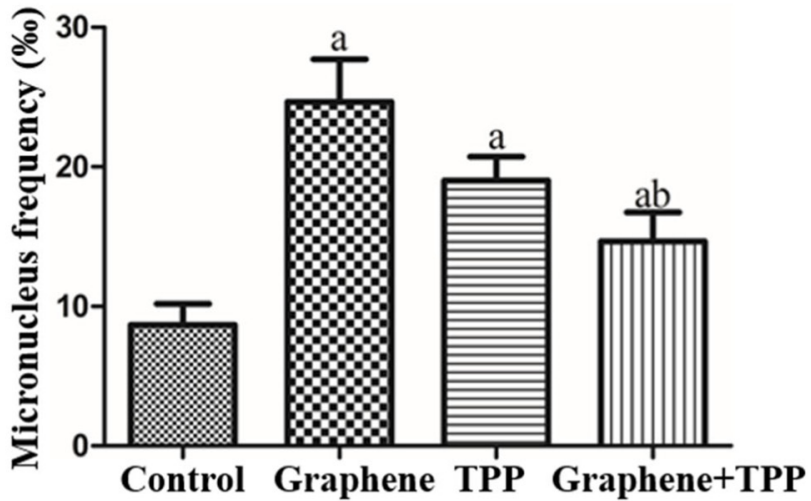

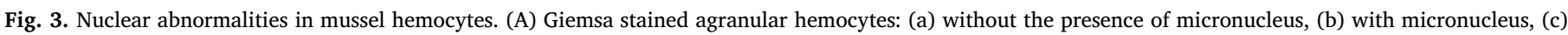

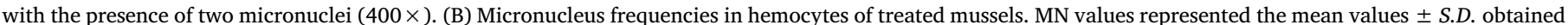
after analysis of samples derived from 15 individuals of each group of mussels.
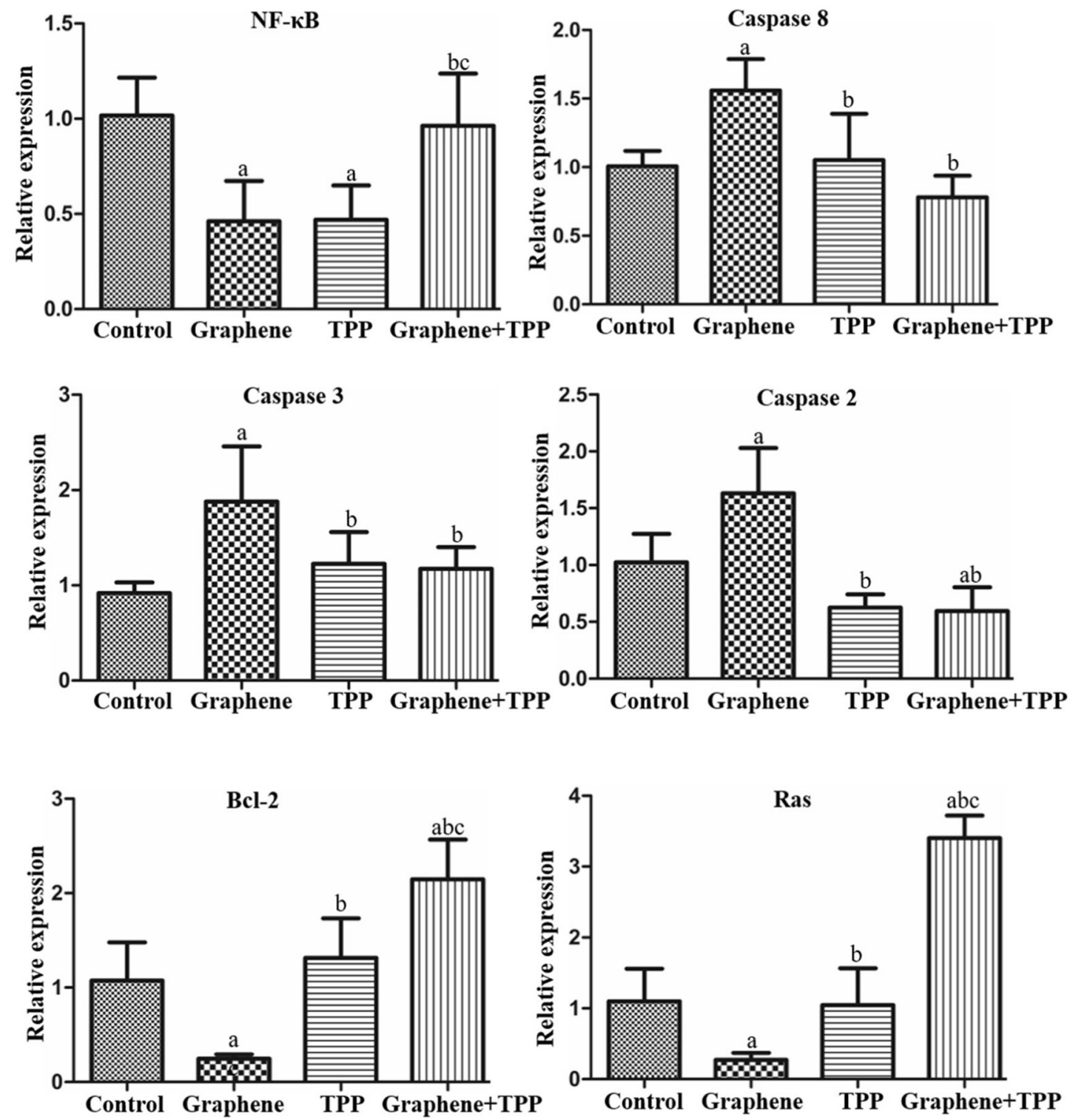

Fig. 4. Relative expressions of genes in the hemocyte of $M$. galloprovincialis after a $7 \mathrm{~d}$ exposure. ${ }^{\mathrm{a}} P<0.05$, compared with the control group; ${ }^{\mathrm{b}} P<0.05$, compared with the graphene-exposed group; ${ }^{\mathrm{c}} P<0.05$, compared with the TPP-exposed group. 

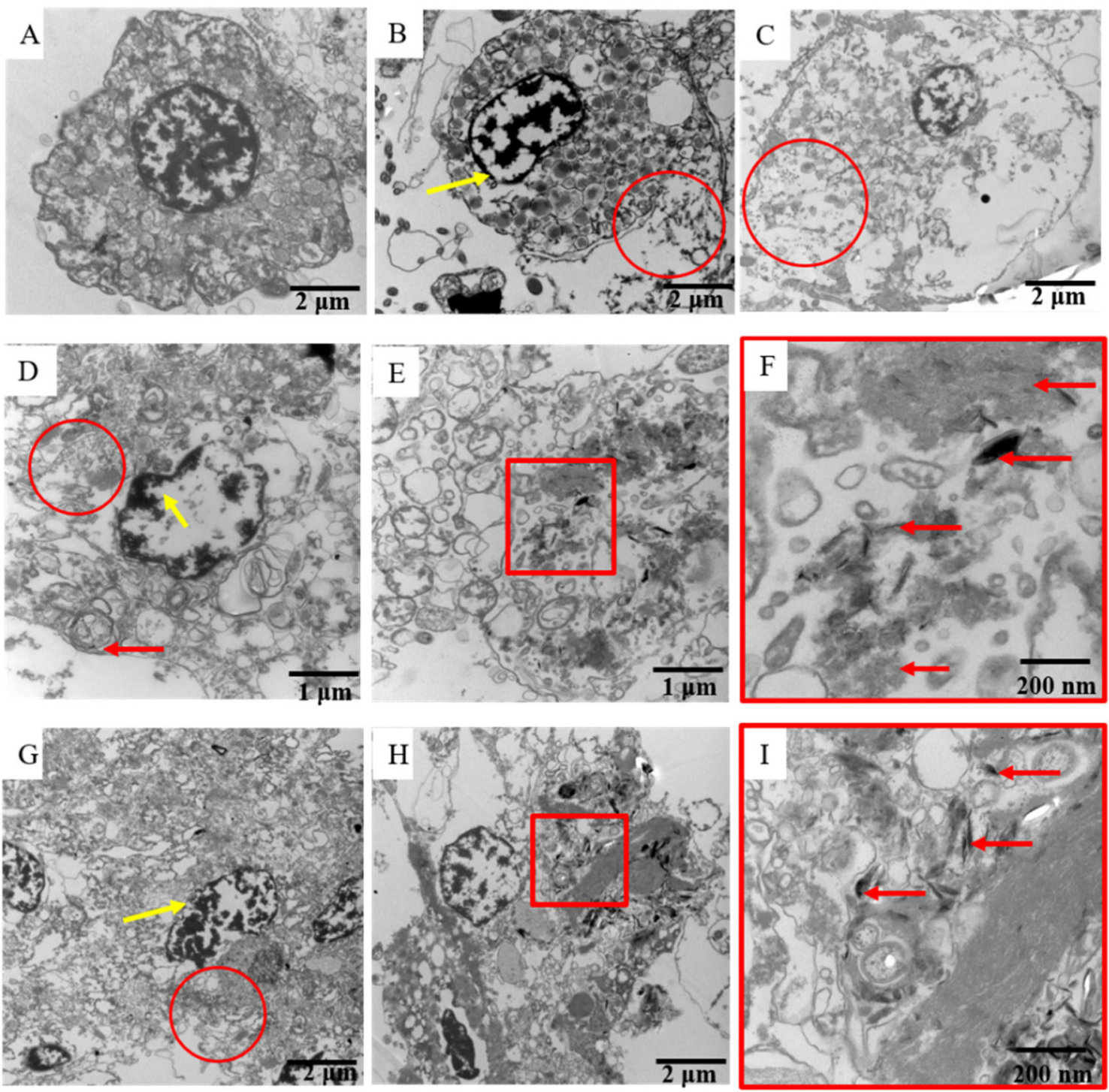

Fig. 5. TEM analysis of hemocytes. (A) Control cells, (B and C) cells exposed to TPP, (D-F) cells exposed to graphene and (G-H) cells exposed to graphene + TPP. Enlarged images (right) from the line squares (red) indicated the location of graphene in cytosol, which were denoted by red arrows. (For interpretation of the references to colour in this figure legend, the reader is referred to the web version of this article.)

Subsequently, caspase 3 and its downstream effector proteins were activated, and apoptosis was initiated (Liang et al., 2019), resulting in the up-regulated expression level of caspase 3 and elevated apoptosis rate in mussel hemocytes. $\mathrm{Bcl}-2$ is involved in many cellular functions such as immune responses, differentiation and block apoptosis (Bruey et al., 2007). Ras can inhibit apoptosis through the activation of $N F-\kappa B$ (Wang et al., 2011). In this work, the expression levels of the apoptotic-related genes (Fig. 4) presented the same tendency as the apoptosis rates (Fig. 2D).

In addition, $N F-\kappa B$ is a pleiotropic transcription factor in almost all cell types and a major regulator of immunity and inflammatory responses to injuries and infections (Napetschnig and Wu, 2013). The relative expression of $N F-\kappa B$ gene in graphene + TPP treatment was evidently up-regulated in comparison with that of graphene-exposed group (Fig. 4). This suggested that the graphene + TPP exposure could induce immune effects through activating $N F-\kappa B$, and then reduce the cytotoxicity caused by graphene in hemocytes.

\subsection{TEM analysis}

The alterations in the cellular ultrastructure and the uptake of graphene were detected by TEM (Fig. 5). As shown in Fig. 5A, there were intact cell membrane, nuclear and other cytoplasmic compartments in control group mussels hemocytes. After exposure to graphene and graphene + TPP, graphene was shown as black irregular structures in transverse sections (Fig. 5D-I) (Zhu et al., 2017), with variable thicknesses depending on the number of layers. Graphene was localized freely in the cytosol (red arrows, Fig. 5D, F and I). In all treatments, cellular membrane damage was observed in mussel hemocytes, such as perforation (red circles, Fig. 5B, D and G) and nuclear membrane thickening and edema (yellow arrows, Fig. 5B, D and G).

The uptake of carbon nanomaterials by cells was reported in a few studies (Oh et al., 2010; Raffa et al., 2010). In this work, graphene was found in hemocytes by TEM investigation (Fig. 5). Katsumiti et al. (2017) also found that GO could be accumulated in the cytosol and in endolysosomal vesicles of M. galloprovincialis hemocytes. After internalization, the accumulation of graphene in cell cytoplasm, perinuclear space, and nucleus could induce cytotoxicity by triggering apoptosis through activation of mitochondrial pathway and increase oxidative stress through depletion of the mitochondrial membrane potential ( $\mathrm{Li}$ et al., 2012). Therefore, it was evidenced that both graphene and graphene + TPP could induce oxidative stress and alter the antioxidant 


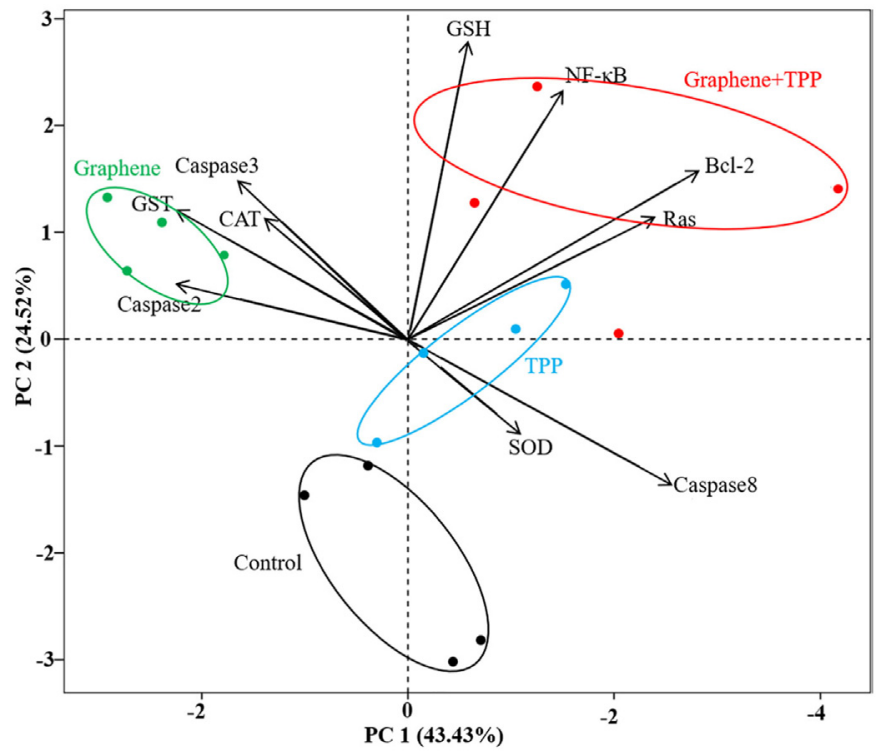

Fig. 6. IBR star plots of the biomarkers in $M$. galloprovincialis for four different treatments. (A) Presented the response of the six biomarkers (MN, LMS, CAT, SOD, GSH and GST). (B) Showed the whole biomarker IBR values for each treatment. $\mathrm{MN}$ = DNA damage; LMS = lysosomal membrane stability; SOD = superoxide dismutase; $\mathrm{CAT}=$ catalase; $\mathrm{GSH}=$ glutathione; GST = glutathione-s-transferase (black line: control, green line: graphene-exposed group, blue line: TPP-exposed group, red line: graphene + TPP-exposed group). (For interpretation of the references to colour in this figure legend, the reader is referred to the web version of this article.)

enzyme activities in mussels (Table 2).

\subsection{Integrated biomarker indices}

IBR index has been widely applied to measure ecosystem health and identified influences of environmental stress on organisms (Turja et al., 2014). The IBR approach is frequently employed to explore both native and caged bivalves (Campillo et al., 2013; Marigomez et al., 2013). The star plots of IBR presented the response of the six biomarkers (MN, LMS, CAT, SOD, GSH and GST) (Fig. 6A). IBR values in the M. galloprovincialis from graphene, TPP and graphene + TPP treatments were 24, 19 and
21, respectively (Fig. 7). The IBR value in graphene + TPP treatment was lower than that in graphene treatment, reflecting that graphene + TPP induced lower toxicities on M. galloprovincialis hemocytes than the single exposure of graphene. This was consistent with the findings in antioxidant status and cytotoxicity, as mentioned above.

\section{Conclusion}

This study demonstrated that graphene and TPP could induce intracellular oxidative stress and cytotoxicity in mussel hemocytes. The graphene internalized into the hemocytes, which further contributed to the oxidative stress. The oxidative stress and DNA damage were reduced in the combined graphene and TPP-treated group, comparing with those in the graphene-exposed group. In addition, the genes of immunity and block apoptosis ( $N F-\kappa B, B c l-2$ and Ras) were significantly up-regulated in graphene + TPP-exposed group, which was mainly associated with reduced apoptosis and DNA damage. IBR values also showed that the graphene + TPP exposure induced lower toxicities than graphene. In conclusion, the combined exposure of graphene and TPP could reduce cytotoxicity caused by the single exposure of graphene in the hemocytes of M. galloprovincialis (Fig. 7).

\section{Authors' contributions}

Xiangjing Meng conducted the main experiments and drafted the manuscript; Fei Li and Huifeng Wu designed the experiments, directed the project and provided major revisions; Xiaoqing Wang, Jialin Liu and Chenglong Ji interpreted the data and revised the manuscript; all authors contributed to discussions. All authors read and approved the final manuscript.

\section{Declaration of competing interest}

The authors declare that they have no known competing financial interests or personal relationships that could have appeared to influence the work reported in this paper.

\section{Acknowledgements}

This research was supported by the National Natural Science Foundation of China (41530642, 21677173, 41976152) and the Youth Innovation Promotion Association CAS (2017255).
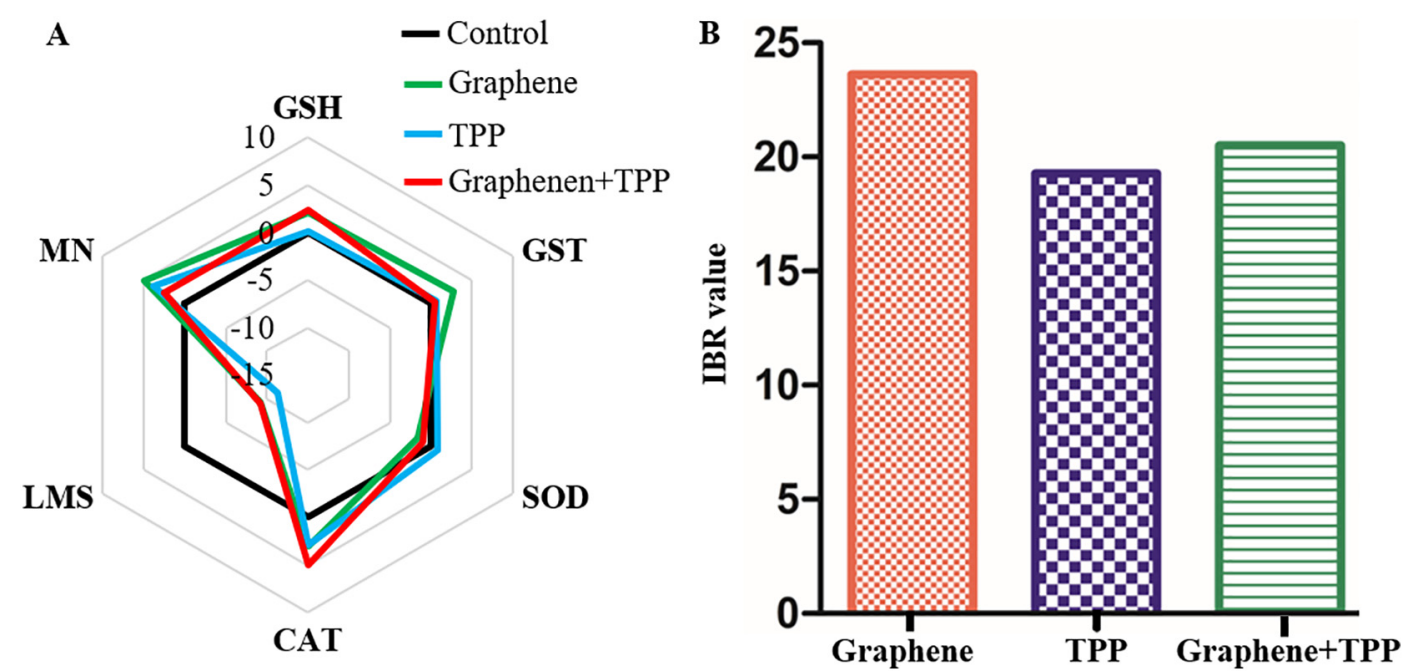

Fig. 7. IBR star plots of the biomarkers in M. galloprovincialis for four different treatments. (A) Present the response of the six biomarkers (MN, LMS, CAT, SOD, GSH and GST). (B) Shows the whole biomarker IBR values for each treatment. MN = DNA damage; LMS = lysosomal membrane stability; SOD = superoxide dismutase; CAT = catalase; GSH = glutathione; GST = glutathione-s-transferase (black line: control, green line: graphene, blue line: TPP, red line: graphene + TPP). (For interpretation of the references to colour in this figure legend, the reader is referred to the web version of this article.) 


\section{References}

Al-Subiai, S., Arlt, V., Frickers, P., Readman, J., Stolpe, B., Lead, J., Moody, A., Jha, A., 2012. Merging nano-genotoxicology with eco-genotoxicology: an integrated approach to determine interactive genotoxic and sub-lethal toxic effects of C60 fullerenes and fluoranthene in marine mussels, Mytilus sp. Mutat. Res. Genet. Toxicol. Environ. Mutagen. 745 (1-2), 92-103.

Balbi, T., Smerilli, A., Fabbri, R., Ciacci, C., Montagna, M., Grasselli, E., Brunelli, A., Pojana, G., Marcomini, A., Gallo, G., 2014. Co-exposure to $\mathrm{n}-\mathrm{TiO}_{2}$ and $\mathrm{Cd}^{2+}$ results in interactive effects on biomarker responses but not in increased toxicity in the marine bivalve M. galloprovincialis. Sci. Total Environ. 15 (493), 355-364.

Balbi, T., Ciacci, C., Grasselli, E., Smerilli, A., Voci, A., Canesi, L., 2017. Utilization of Mytilus digestive gland cells for the in vitro screening of potential metabolic disruptors in aquatic invertebrates. Comp. Biochem. Physiol. C Toxicol. Pharmacol. 191, 26-35.

Balbi, T., Cortese, K., Ciacci, C., Bellese, G., Vezzulli, L., Pruzzo, C., Canesi, L., 2018. Autophagic processes in Mytilus galloprovincialis hemocytes: effects of Vibrio tapetis. Fish Shellfish Immunol 73, 66-74.

Beliaeff, B., Burgeot, T., 2002. Integrated biomarker response: a useful tool for ecological risk assessment. Environ. Toxicol. Chem. 21 (6), 1316-1322.

Bolognesi, C., Fenech, M., 2012. Mussel micronucleus cytome assay. Nat. Protoc. 7 (6), $1125-1137$.

Brandts, I., Teles, M., Goncalves, A.P., Barreto, A., Franco-Martinez, L., Tvarijonaviciute, A., Martins, M.A., Soares, A., Tort, L., Oliveira, M., 2018. Effects of nanoplastics on Mytilus galloprovincialis after individual and combined exposure with carbamazepine. Sci. Total Environ. 643, 775-784.

Bruey, J., Bruey-Sedano, N., Luciano, F., Zhai, D., Balpai, R., Xu, C., Kress, C., BaillyMaitre, B., Li, X., Osterman, A., Matsuzawa, S., Terskikh, A., Faustin, B., Reed, J., 2007. Bcl-2 and Bcl-XL regulate proinflammatory caspase- 1 activation by interaction with NALP1. Cell 129 (1), 45-56.

Campillo, J., Albentosa, M., Valdes, N., Moreno-Gonzalez, R., Leon, V., 2013. Impact assessment of agricultural inputs into a Mediterranean coastal lagoon (Mar Menor, SE Spain) on transplanted clams (Ruditapes decussatus) by biochemical and physiological responses. Aquat. Toxicol. 15 (142-143), 365-379.

Canesi, L., Corsi, I., 2016. Effects of nanomaterials on marine invertebrates. Sci. Total Environ. 565, 933-940.

Canesi, L., Ciacci, C., Betti, M., Fabbri, R., Canonico, B., Fantinati, A., Marcornini, A., Pojana, G., 2008. Immunotoxicity of carbon black nanoparticles to blue mussel hemocytes. Environ. Int. 34 (8), 1114-1119.

Canesi, L., Ciacci, C., Fabbri, R., Marcomini, A., Pojana, G., Gallo, G., 2012. Bivalve molluscs as a unique target group for nanoparticle toxicity. Mar. Environ. Res. 76, $16-21$.

Canesi, L., Ciacci, C., Bergami, E., Monopoli, M., Dawson, K., Papa, S., Canonico, B., Corsi, I., 2015. Evidence for immunomodulation and apoptotic processes induced by cationic polystyrene nanoparticles in the hemocytes of the marine bivalve Mytilus. Mar. Environ. Res. 111, 34-40.

Canty, M., Hutchinson, T., Brown, R., Jones, M., Jha, A., 2009. Linking genotoxic responses with cytotoxic and behavioural or physiological consequences: differential sensitivity of echinoderms (Asterias rubens) and marine molluscs (Mytilus edulis). Aquat. Toxicol. 94 (1), 68-76.

Cao, R., Liu, Y., Wang, Q., Dong, Z., Yang, D., Liu, H., Ran, W., Qu, Y., Zhao, J., 2018. Seawater acidification aggravated cadmium toxicity in the oyster Crassostrea gigas: metal bioaccumulation, subcellular distribution and multiple physiological responses. Sci. Total Environ. 642, 809-823.

Chen, G., Jin, Y., Wu, Y., Liu, L., Fu, Z., 2015. Exposure of male mice to two kinds of organophosphate flame retardants (OPFRs) induced oxidative stress and endocrine disruption. Environ. Toxicol. Pharmacol. 40 (1), 310-318.

Chowdhury, I., Duch, M., Mansukhani, N., Hersam, M., Bouchard, D., 2013. Colloidal properties and stability of graphene oxide nanomaterials in the aquatic environment. Environ. Sci. Technol. 47, 6288-6296.

Coteur, G., Danis, B., Wantier, P., Pernet, P., Dubois, P., 2005. Increased phagocytic activity in contaminated seastars (Asterias rubens) collected in the southern bight of the North Sea. Mar. Pollut. Bull. 50 (11), 1295-1302.

Dallas, L., Jha, A., 2015. Applications of biological tools or biomarkers in aquatic biota: a case study of the Tamar estuary, South West England. Mar. Pollut. Bull. 95 (2), 618-633.

Della Torre, C., Balbi, T., Grassi, G., Frenzilli, G., Bernardeschi, M., Smerilli, A., Guidi, P., Canesi, L., Nigro, M., Monaci, F., Scarcelli, V., Rocco, L., Focardi, S., Monopoli, M., Corsi, I., 2015. Titanium dioxide nanoparticles modulate the toxicological response to cadmium in the gills of Mytilus galloprovincialis. J. Hazard. Mater. 297, 92-100.

Di, Y., Schroeder, D., Highfield, A., Readman, J., Jha, A., 2011. Tissue-specific expression of p53 and ras genes in response to the environmental genotoxicant benzo(alpha) pyrene in marine mussels. Environ. Sci. Technol. 45 (20), 8974-8981.

Ding, K., Kong, X., Wang, J., Lu, L., Zhou, W., Zhan, T., Zhang, C., Zhuang, S., 2017. Side chains of parabens modulate antiandrogenic activity: In vitro and molecular docking studies. Environ. Sci. Technol. 51 (11), 6452-6460.

Ellis, R., Parry, H., Spicer, J., Hutchinson, T., Pipe, R., Widdicombe, S., 2011. Immunological function in marine invertebrates: responses to environmental perturbation. Fish Shellfish Immunol 30 (6), 1209-1222.

Glomstad, B., Altin, D., Sørensen, L., Liu, J., Jenssen, B., Booth, A., 2016. Carbon nanotube properties influence adsorption of phenanthrene and subsequent bioavailability and toxicity to Pseudokirchneriella subcapitata. Environ. Sci. Technol. 50 (5), 2660-2668.

Gomes, T., Pereira, C., Cardoso, C., Bebianno, M., 2013. Differential protein expression in mussels Mytilus galloprovincialis exposed to nano and ionic Ag. Aquat. Toxicol. 136-137, 79-90.
Hong, X., Chen, R., Hou, R., Yuan, L., Zha, J., 2018. Triphenyl phosphate (TPHP)-induced neurotoxicity in adult male Chinese rare minnows (Gobiocypris rarus). Environ. Sci. Technol. 52 (20), 11895-11903.

Hu, X., Zhou, Q., 2013. Health and ecosystem risks of graphene. Chem. Rev. 113 (5), 3815-3835.

Hu, X., Kang, J., Lu, K., Zhou, R., Mu, L., Zhou, Q., 2014. Graphene oxide amplifies the phytotoxicity of arsenic in wheat. Sci. Rep. 4, 6122.

Hu, X., Ouyang, S., Mu, L., An, J., Zhou, Q., 2015. Effects of graphene oxide and oxidized carbon nanotubes on the cellular division, microstructure, uptake, oxidative stress, and metabolic profiles. Environ. Sci. Technol. 49, 10825-10833.

Hu, C., Hu, N., Li, X., Zhao, Y., 2016. Graphene oxide alleviates the ecotoxicity of copper on the freshwater microalga Scenedesmus obliquus. Ecotoxicol. Environ. Saf. 132, $360-365$.

Iavicoli, I., Leso, V., Beezhold, D., Shvedova, A., 2017. Nanotechnology in agriculture: opportunities, toxicological implications, and occupational risks. Toxicol. Appl. Pharmacol. 329, 96-111.

Jiang, Y., Tang, X., Sun, T., Wang, Y., 2017. BDE-47 exposure changed the immune function of haemocytes in Mytilus edulis: An explanation based on ROS-mediated pathway. Aquat. Toxicol. 182, 58-66.

Katsumiti, A., Tomovska, R., Cajaraville, M., 2017. Intracellular localization and toxicity of graphene oxide and reduced graphene oxide nanoplatelets to mussel hemocytes in vitro. Aquat. Toxicol. 188, 138-147.

Kim, S., Jung, J., Lee, I., Jung, D., Youn, H., Choi, K., 2015. Thyroid disruption by triphenyl phosphate, an organophosphate flame retardant, in zebrafish (Danio rerio) embryos/larvae, and in GH3 and FRTL-5 cell lines. Aquat. Toxicol. 160, 188-196.

Kolarevic, S., Kracun-Kolarevic, M., Kostic, J., Slobodnik, J., Liska, I., Gacic, Z., Paunovic, M., Knezevic-Vukcevic, J., Vukovic-Gacic, B., 2016. Assessment of the genotoxic potential along the Danube River by application of the comet assay on haemocytes of freshwater mussels: the Joint Danube Survey 3. Sci. Total Environ. 540, 377-385.

Koutsogiannaki, S., Franzellitti, S., Kalogiannis, S., Fabbri, E., Dimitriadis, V., Kaloyianni, M., 2015. Effects of cadmium and 17ß-estradiol on Mytilus galloprovincialis redox status. Prooxidant-antioxidant balance (PAB) as a novel approach in biomonitoring of marine environments. Mar. Environ. Res. 103, 80-88.

Lanphere, J., Rogers, B., Luth, C., Bolster, C., Walker, S., 2014. Stability and transport of graphene oxide nanoparticles in groundwater and surface water. Environ. Eng. Sci. 31, 350-359.

Li, Y., Liu, Y., Fu, Y., Wei, T., Le Guyader, L., Gao, G., Liu, R., Chang, Y., Chen, C., 2012. The triggering of apoptosis in macrophages by pristine graphene through the MAPK and TGF-beta signaling pathways. Biomaterials 33 (2), 402-411.

Li, F., Ding, J., Wu, H., Meng, X., Zhao, J., 2017a. Interaction between graphene joint triphenyl phosphate (TPP) and the simulated phospholipid bilayer of electrochemical research. Chin. Sci. Bull. 62 (36), 4285-4293.

Li, Y., Zhang, L., Qu, T., Tang, X., Li, L., Zhang, G., 2017b. Conservation and divergence of mitochondrial apoptosis pathway in the Pacific oyster, Crassostrea gigas. Cell Death Dis. 8 (7), e2915.

Liang, T., Xu, X., Ye, D., Chen, W., Gao, B., Huang, Y., 2019. Caspase/AIF/apoptosis pathway: a new target of puerarin for diabetes mellitus therapy. Mol. Biol. Rep. 1-11.

Liu, X., Ji, K., Choi, K., 2012. Endocrine disruption potentials of organophosphate flame retardants and related mechanisms in H295R and MVLN cell lines and in zebrafish. Aquat. Toxicol. 114-115, 173-181.

Liu, S., Jiang, W., Wu, B., Yu, J., Yu, H., Zhang, X., Torres-Duarte, C., Cherr, G., 2016. Low levels of graphene and graphene oxide inhibit cellular xenobiotic defense system mediated by efflux transporters. Nanotoxicology 10 (5), 597-606.

Livak, K., Schmittgen, T., 2001. Analysis of relative gene expression data using real-time quantitative PCR and the 2(T) (-Delta Delta C) method. Methods 25 (4), 402-408.

Marigomez, I., Zorita, I., Izagirre, U., Ortiz-Zarragoitia, M., Navarro, P., Etxebarria, N., Orbea, A., Soto, M., Cajaraville, M., 2013. Combined use of native and caged mussels to assess biological effects of pollution through the integrative biomarker approach. Aquat. Toxicol. 136, 32-48.

Matozzo, V., Battistara, M., Marisa, I., Bertin, V., Orsetti, A., 2016. Assessing the effects of amoxicillin on antioxidant enzyme activities, lipid peroxidation and protein carbonyl content in the clam Ruditapes philippinarum and the mussel Mytilus galloprovincialis. Bull. Environ. Contam. Toxicol. 97 (4), 521-527.

Meng, X., Li, F., Wang, X., Liu, J., Ji, C., Wu, H., 2019. Combinatorial immune and stress response, cytoskeleton and signal transduction effects of graphene and triphenyl phosphate (TPP) in mussel Mytilus galloprovincialis. J. Hazard. Mater. 378, 120778.

Mezzelani, M., Gorbi, S., Da Ros, Z., Fattorini, D., d'Errico, G., Milan, M., Bargelloni, L., Regoli, F., 2016. Ecotoxicological potential of non-steroidal anti-inflammatory drugs (NSAIDs) in marine organisms: bioavailability, biomarkers and natural occurrence in Mytilus galloprovincialis. Mar. Environ. Res. 121, 31-39.

Mittal, S., Kumar, V., Dhiman, N., Chauhan, L.K., Pasricha, R., Pandey, A., 2016. Physicochemical properties based differential toxicity of graphene oxide/reduced graphene oxide in human lung cells mediated through oxidative stress. Sci. Rep. 21 (6), 39548-39563.

Moore, M., 2006. Do nanoparticles present ecotoxicological risks for the health of the aquatic environment? Environ. Int. 32 (8), 967-976.

Moore, M., 2008. Autophagy as a second level protective process in conferring resistance to environmentally-induced oxidative stress. Autophagy 4 (2), 254-256.

Napetschnig, J., Wu, H., 2013. Molecular basis of NF-kB signaling. Annu. Rev. Biophys. 42, 443-468.

Oh, W.K., Kim, S., Yoon, H., Jang, J., 2010. Shape-dependent cytotoxicity and proinflammatory response of poly (3,4-ethylenedioxythiophene) nanomaterials. Small 6 (7), 872-879.

Ou, L., Song, B., Liang, H., Liu, J., Feng, X., Deng, B., Sun, T., Shao, L., 2016. Toxicity of graphene-family nanoparticles: a general review of the origins and mechanisms. Part. Fibre Toxicol. 13 (1), 57-81. 
Pipe, R., Coles, J., Carissan, F., Ramanathan, K., 1999. Copper induced immunomodulation in the marine mussel, Mytilus edulis. Aquat. Toxicol. 46 (1), 43-54.

Politakis, N., Belavgeni, A., Efthimiou, I., Charalampous, N., Kourkouta, C., Dailianis, S., 2018. The impact of expired commercial drugs on non-target marine species: a case study with the use of a battery of biomarkers in hemocytes of mussels. Ecotoxicol. Environ. Saf. 148, 160-168.

Pretti, C., Oliva, M., Di Pietro, R., Monni, G., Cevasco, G., Chiellini, F., Pomelli, C. Chiappe, C., 2014. Ecotoxicity of pristine graphene to marine organisms. Ecotoxicol. Environ. Saf. 101, 138-145.

Qiu, Y., Wang, Z., Owens, A., Kulaots, I., Chen, Y., Kane, A., Hurt, R., 2014. Antioxidant chemistry of graphene-based materials and its role in oxidation protection technology. Nanoscale 6 (20), 11744-11755.

Raffa, V., Ciofani, G., Vittorio, O., Riggio, C., Cuschieri, A., 2010. Physicochemical properties affecting cellular uptake of carbon nanotubes. Nanomedicine (Lond) 5 (1), 89-97.

Romero, A., Novoa, B., Figueras, A., 2015. The complexity of apoptotic cell death in mollusks: An update. Fish Shellfish Immunol 46 (1), 79-87.

Sun, H., Feng, R., Nan, Y., Chen, Z., Sang, N., 2018. In-situ examination of graphene and graphene oxide impact on the depuration of phenanthrene and fluoranthene adsorbed onto spinach (Spinacia oleracea L.) leaf surfaces. Environ. Pollut. 237, 968-976.

Turja, R., Hoher, N., Snoeijs, P., Barsiene, J., Butrimaviciene, L., Kuznetsova, T., Kholodkevich, S., Devier, M., Budzinski, H., Lehtonen, K., 2014. A multibiomarker approach to the assessment of pollution impacts in two Baltic Sea coastal areas in Sweden using caged mussels (Mytilus trossulus). Sci. Total Environ. 473, 398-409.

Vallabani, N., Mittal, S., Shukla, R., Pandey, A., Dhakate, S., Pasricha, R., Dhawan, A., 2011. Toxicity of graphene in normal human lung cells (BEAS-2B). J. Biomed.
Nanotechnol. 7 (1), 106-107.

Van der Veen, I., De Boer, J., 2012. Phosphorus flame retardants: properties, production, environmental occurrence, toxicity and analysis. Chemosphere 88 (10), 1119-1153.

Wang, Z., Banerjee, S., Ahmad, A., Li, Y., Azmi, A., Gunn, J., Kong, D., Bao, B., Ali, S. Gao, J., 2011. Activated K-ras and INK4a/Arf deficiency cooperate during the development of pancreatic cancer by activation of Notch and NF-kB signaling pathways. PLoS One 6 (6), e20537.

Wu, X., Wang, W., Zhu, L., 2018. Enhanced organic contaminants accumulation in crops: mechanisms, interactions with engineered nanomaterials in soil. Environ. Pollut. 240, 51-59.

Xie, J., Zhao, Y., Wang, Q., Wu, H., Teng, J., Yang, D., Cao, I., Chen, L., Li, F., Ji, C., Cong, M., Zhao, J., 2016. An integrative biomarker approach to assess the environmental stress in the north coast of Shandong Peninsula using native oysters, Crassostrea gigas. Mar. Pollut. Bull. 112 (1-2), 318-326.

Zhang, Y., Chen, S., Qu, M., Adeleye, A., Di, Y., 2017a. Utilization of isolated marine mussel cells as an in vitro model to assess xenobiotics induced genotoxicity. Toxicol. in Vitro 44, 219-229.

Zhang, X., Zhou, Q., Zou, W., Hu, X., 2017b. Molecular mechanisms of developmental toxicity induced by graphene oxide at predicted environmental concentrations. Environ. Sci. Technol. 51 (14), 7861-7871.

Zhong, M., Wu, H., Mi, W., Li, F., Ji, C., Ebinghaus, R., Tang, J., Xie, Z., 2018. Occurrences and distribution characteristics of organophosphate ester flame retardants and plasticizers in the sediments of the Bohai and Yellow Seas, China. Sci. Total Environ. 615, 1305-1311.

Zhu, S., Luo, F., Chen, W., Zhu, B., Wang, G., 2017. Toxicity evaluation of graphene oxide on cysts and three larval stages of Artemia salina. Sci. Total Environ. 595, 101-109. 